\title{
Evaluating the effect of diabetes on the prognosis of gastric cancer using a propensity score matching method
}

\author{
Lili Sheng ${ }^{1 *}$, Hui Peng ${ }^{1 *}$, Yang Pan ${ }^{2}$, Chengci Wang ${ }^{2}$, Yiping Zhu ${ }^{1}$ \\ ${ }^{1}$ Department of Oncology, the First Affiliated Hospital of Wannan Medical College, Wuhu, China; ${ }^{2}$ Wannan Medical College, Wuhu, China \\ Contributions: (I) Conception and design: L Sheng; (II) Administrative support: C Wang; (III) Provision of study materials or patients: Y Pan, Y Zhu; \\ (IV) Collection and assembly of data: H Peng; (V) Data analysis and interpretation: L Sheng, H Peng; (VI) Manuscript writing: All authors; (VII) \\ Final approval of manuscript: All authors. \\ "These authors contributed equally to this work. \\ Correspondence to: Yiping Zhu. Department of Oncology, the First Affiliated Hospital of Wannan Medical College, Wuhu, China. \\ Email: zhuyiping@wnmc.edu.cn.
}

Background: Gastric cancer (GC) is one of the malignant tumors with high incidence in China. At present, the relationship between type 2 diabetes (T2DM) and the therapeutic effect of various malignant tumors has attracted more and more attention. This study aimed to investigate whether T2DM is a prognostic factor for patients with GC.

Methods: Patients who had GC and who were admitted to our hospital from November 2008 to December 2015 were included in the study. Among these patients, 84 patients GC complicated with T2DM (GC + T2DM) were enrolled in the observation group, and 215 patients with normal blood glucose were enrolled in the control group. Patients' general information was collected by referring to their electronic and paper medical records, and their living status was followed up by conducting a telephone survey, referring to their hospitalization record, and performing an outpatient review. A propensity score matching method was used to select a 1:1 matched control for each patient with GC and diabetes. An overall survival curve was established using the Kaplan-Meier method. The survival rate was compared via a log-rank test. A Cox proportional hazards regression model was used to the analyse single and multiple factors affecting patient outcomes.

Results: Before matching was conducted, the differences in gender, stage, treatment, and comorbidity were found to be statistically significant $(\mathrm{P}>0.05)$. After matching was completed, the clinical data and pathological differences between the two groups were not statistically significant $(\mathrm{P}<0.05)$. A histogram matching the preand post-propensity scores showed that the matching was successful. The results of the Cox regression model revealed that grouping, pathological type, and treatment were the independent risk factors of the survival of patients with GC. Survival analysis found that the 3-year, 5-year, and overall survival rates of the observation group were significantly lower than those of the control group $(\mathrm{P}<0.05)$.

Conclusions: T2DM plays an important role in the development of GC, and is a prognostic factor among patients with GC.

Keywords: Gastric cancer (GC); type 2 diabetes mellitus (T2DM); prognosis; propensity score matching method

Submitted Aug 10, 2020. Accepted for publication Sep 30, 2020.

doi: 10.21037/jgo-20-375

View this article at: http://dx.doi.org/10.21037/jgo-20-375 


\section{Introduction}

According to the latest data from the National Cancer Registry, approximately 769,000 new cases of gastric cancer (GC) were recorded in China, and approximately 498,000 deaths from GC occurred in 2015; hence, GC is a major disease that seriously harms the health of the Chinese population $(1,2)$. The incidence of diabetes has also gradually increased, and it has become the fifth leading cause of death in the world (3). Sekikawa et al. (4) found that type 2 diabetes mellitus (T2DM) can increase the risk of GC. Moreover, the risk of GC in patients with diabetes in China is higher than that of patients without diabetes (5). Diabetes and GC have a close relationship. Patients with diabetes are hyperglycaemic for a prolonged period, making them unpreventably susceptible to Helicobacter pylori infection (6), thus increasing the incidence of GC turn. Other factors that increase the incidence of GC include hypoglycemic drugs, insulin resistance, and hyperinsulinemia. Studies on diabetes and cancer prognosis have confirmed that diabetes increases the risk of death caused by cancers like colorectal cancer and GC $(7,8)$, but whether diabetes is an indicator of a poor prognosis for GC patients remains unclear. This study used a propensitymatching method to match the clinical features of patients with GC and T2DM, balanced the effects of covariates between groups, performed survival analysis on matched data, objectively evaluated the prognostic effect of diabetes, and explored the difference between GC patients with and without diabetes. We present the following article in accordance with the SRTOBE reporting checklist (available at http://dx.doi.org/10.21037/jgo-20-375).

\section{Methods}

\section{Clinical data}

The clinical and pathological data of patients who had GC and were admitted to our hospital from November 2008 to December 2015 were collected. All were diagnosed through clinical pathology. In all, 84 patients with GC and T2DM $(\mathrm{GC}+\mathrm{T} 2 \mathrm{DM})$ were enrolled in the observation group, and 215 patients with normal blood glucose were enrolled in the control group. The diagnostic criteria of T2DM met the T2DM diagnosis and treatment guidelines issued by the American Diabetes Association in 2008. All procedures performed in this study involving human participants were in accordance with the Declaration of Helsinki (as revised in 2013). The study was approved by Scientific Research and New Technology IRB of Wannnan Medical College Yijinshan Hospital. Because of the retrospective nature of the research, the requirement for informed consent was waived.

\section{Research methods}

Patients' general information, including age, gender, comorbidities, pathological types, tumour stage, tumour markers, treatment modalities, and diabetes-related conditions, and data obtained via telephone surveys, hospitalizations and outpatient visits were collected by reviewing their electronic and paper medical records. The patient's survival status was followed up. After the treatment, the patients were followed up once every 3-4 months within the first 2 years, every 4-6 months within 3-5 years, and every 6 months to 1 year after 5 years. Follow-up duration was until December 2018. The primary outcome measurement in this study was overall survival (OS), and the OS time was considered to be from the time of diagnosis to the end of a patient's life or the end of the follow-up period. The secondary outcome measures included 1-, 3-, and 5 -year survival.

\section{Statistical analysis}

Statistical analyses were performed using SPSS 20.0 and $\mathrm{R}$ version 3.0.1. Differences in categorical variables were compared using a C or Fisher's test. Differences in continuous variables were compared using a MannWhitney $\mathrm{U}$ test or a $t$-test. An OS curve was established with the Kaplan-Meier method. The survival rate was compared via a log-rank test. A Cox proportional hazards regression model was used to analyze single and multiple factors affecting the prognosis of patients. A $\mathrm{P}$ value $<0.05$ indicated a statistically significant difference. The propensity score matching method (PSM) was used to select a 1:1 matched comparison for patients with GC and diabetes mellitus $(\mathrm{N}=84)$. The matching factors included age, gender, treatment mode, stage, pathological type, 
Table 1 Comparison of clinical baseline data and pathological features between the two groups before matching

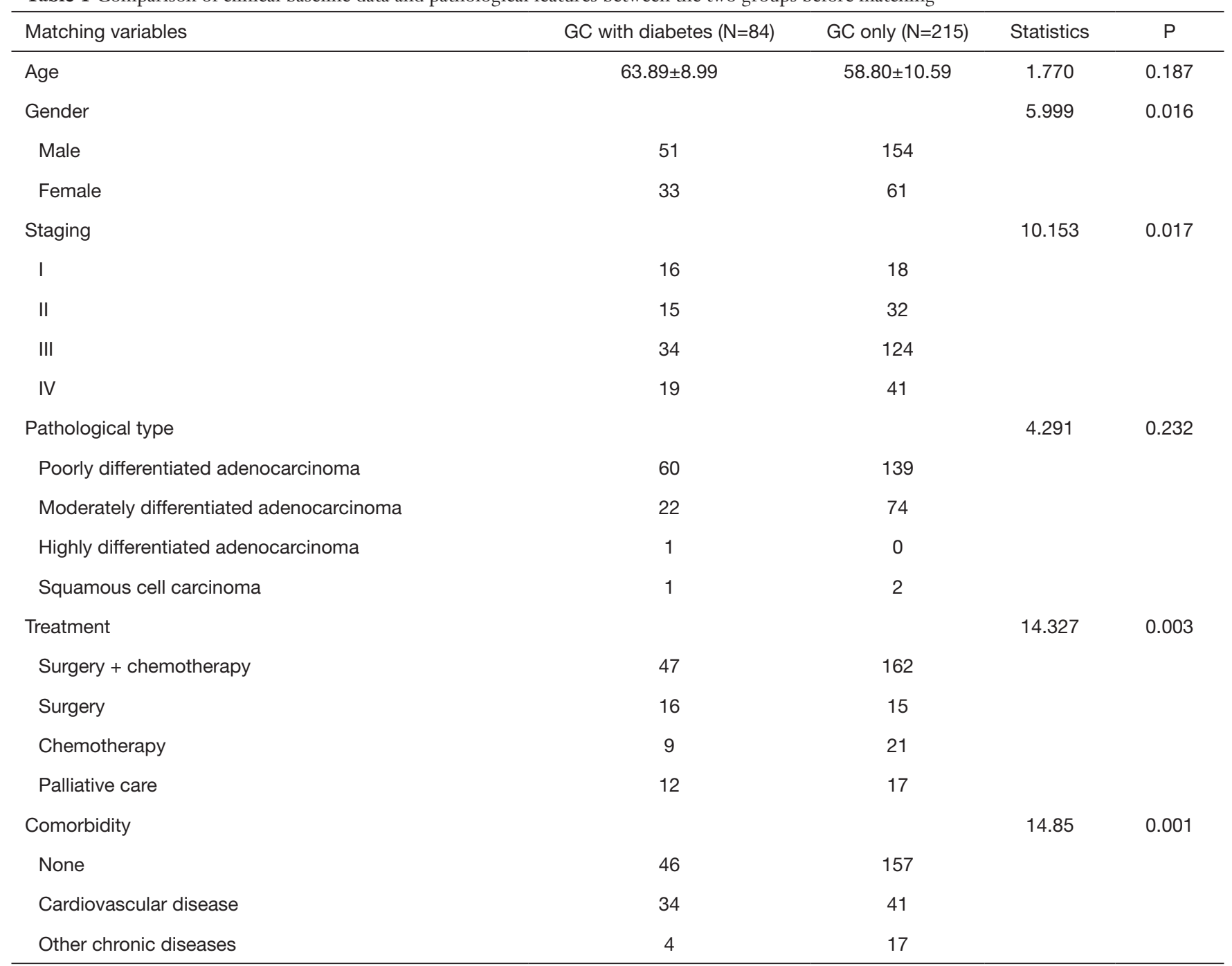

GC, gastric cancer.

and comorbidity. The PSM was implemented using the MatchIt package (version 4.8.3.4) in $\mathrm{R}$ version 3.0.1, and the "nearest" method was used for matching.

\section{Results}

Comparison of clinical data and pathological features between the two groups before and after matching

Before matching was conducted, a statistically significant difference was observed between the two groups in terms of gender, stage, treatment, and comorbidities (Table 1). After matching was completed, the clinical data and pathological differences between the two groups were not statistically significant (Table 2), and the groups were comparable. This observation could also be observed in the histograms of the scores before and after the matching. Figure $1 A, B$ show the histograms before matching. Significant differences were observed between the two groups. Figure 1C,D illustrate the histograms after matching and present two similar sets of graphs. The value and visual data indicated that the matching was successful and that the two matched sets of patient data could be used for subsequent study analysis 
Table 2 Comparison of clinical baseline data and pathological features between the two groups after matching

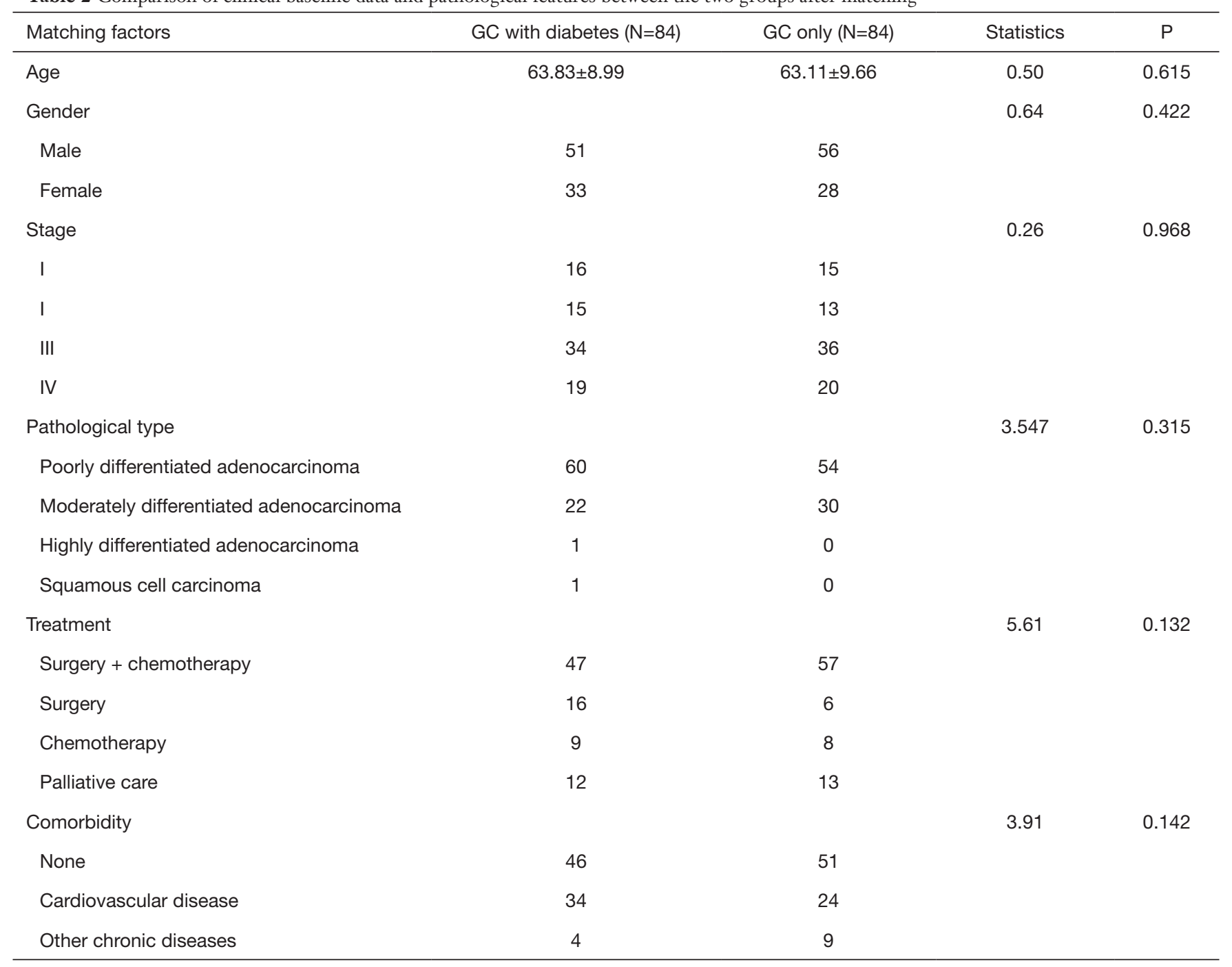

GC, gastric cancer.

(Figure 1).

Cox risk regression model analysis of factors affecting the survival of patients with $G C$

The data concerning the age, gender, grouping, stage, pathological type, treatment, and comorbidities of the patients were subjected to single- and multi-factor analyses. Single-factor analysis showed that grouping, staging, pathological type, treatment, and comorbidities affected the survival of patients with GC. These factors were further analyzed through multi-factor analysis. The results showed that grouping, pathological type and treatment were independent risk factors for survival of GC patients (Table 3).

\section{Survival analysis before and after matching between the two groups of patients}

The two groups of patients were followed up via a telephone interview. The total follow-up time of the 215 patients with simple GC was 112.30 months, and the median follow-up time was 64.60 months. The total follow-up time of the 84 patients with simple GC was 96.53 months, and the median follow-up time was 54.90 months. The total follow-up 


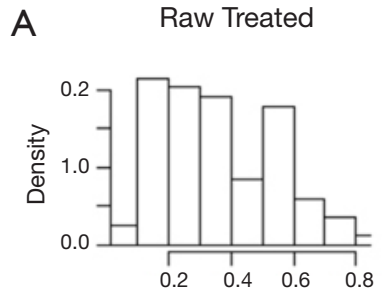

Propensity Score

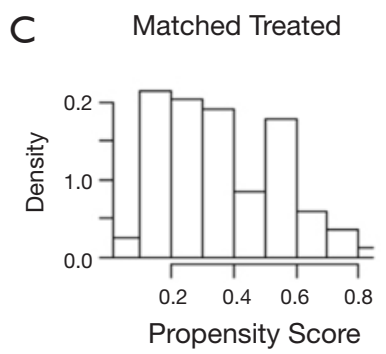

Figure 1 Histograms of propensity scores before and after matching.

time of patients with GC and diabetes was 94.07 months, and the median follow-up time was 28.93 months. The statistical results showed no significant difference in the 1-year survival rate between the two groups before and after the matching $(\mathrm{P}>0.05)$, whereas the 3 - and 5-year survival rates and the $\mathrm{OS}$ rates significantly differed $(\mathrm{P}<0.05$, Table 4, Figures 2,3).

\section{Discussion}

The correlation and interaction mechanisms of diabetes and cancer have been widely explored. A study in China in 2018 showed that T2DM increases the risk of cancer, especially liver cancer, pancreatic cancer and breast cancer, in Chinese people (9). A meta-analysis on 19 Asian cohort studies, including 7 Chinese populations, has shown that T2DM causes a $26 \%$ increase in cancer mortality, including in colorectal cancer, liver cancer, cholangiocarcinoma, gallbladder cancer, pancreatic cancer, and breast cancer $(10,11)$. However, the effect of diabetes on the occurrence, development, and prognosis of GC remains unclear, and no definite conclusion has been made. Three metaanalyses (12-14) have also shown that diabetes increases the incidence and mortality of GC, especially in female patients $(12,13)$. However, the results of a meta-analysis in

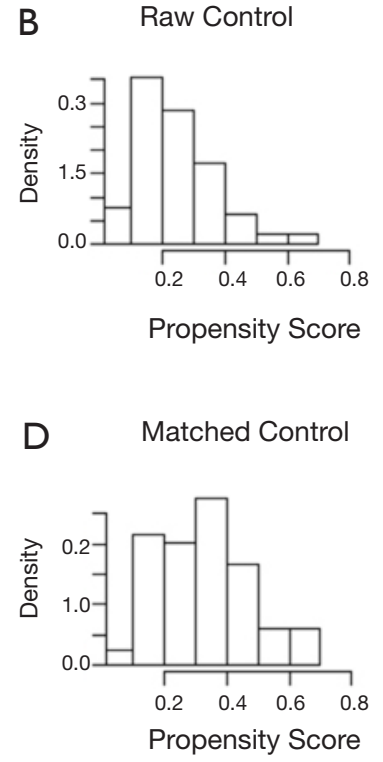

2017 only revealed slight or no change in the risk of death in patients with diabetes mellitus and GC, and no evidence indicates a difference in the relative risk of GC between males and females (15). Therefore, further research on the development of diabetes in GC should be conducted.

The biological mechanism behind the association between diabetes and cancer is unclear, but several aspects of the possible biological mechanism have been hypothesized. (I) For instance, in hyperinsulinemia, the majority of tumour cells express insulin receptor (INS-R) and IGF-1R on their surface. Insulin reduces liverproducing IGF-binding protein (IGFBP1; IGFBP2) and increases free and active IGF1 in circulating blood. When INS-R and IGF-1R bind to the corresponding ligand, they promote the mitosis and metastasis of tumour cells (16). In patients suffering from diabetes and exhibiting insulin resistance, insulin levels in the body increase, and this condition may promote the development of cancer through this pathway. (II) In hyperglycaemia, many tumour cells rely on glycolysis for energy supply, which is highly dependent on glucose, and uncontrolled hyperglycemia is beneficial to cancer cell proliferation (17). (III) Furthermore, in chronic inflammation, T2DM is a chronic low-grade inflammatory disease. T2DM and related obesity can promote the development of malignant tumours. Adipose 
Table 3 COX risk regression model analysis of factors affecting the survival of patients with GC

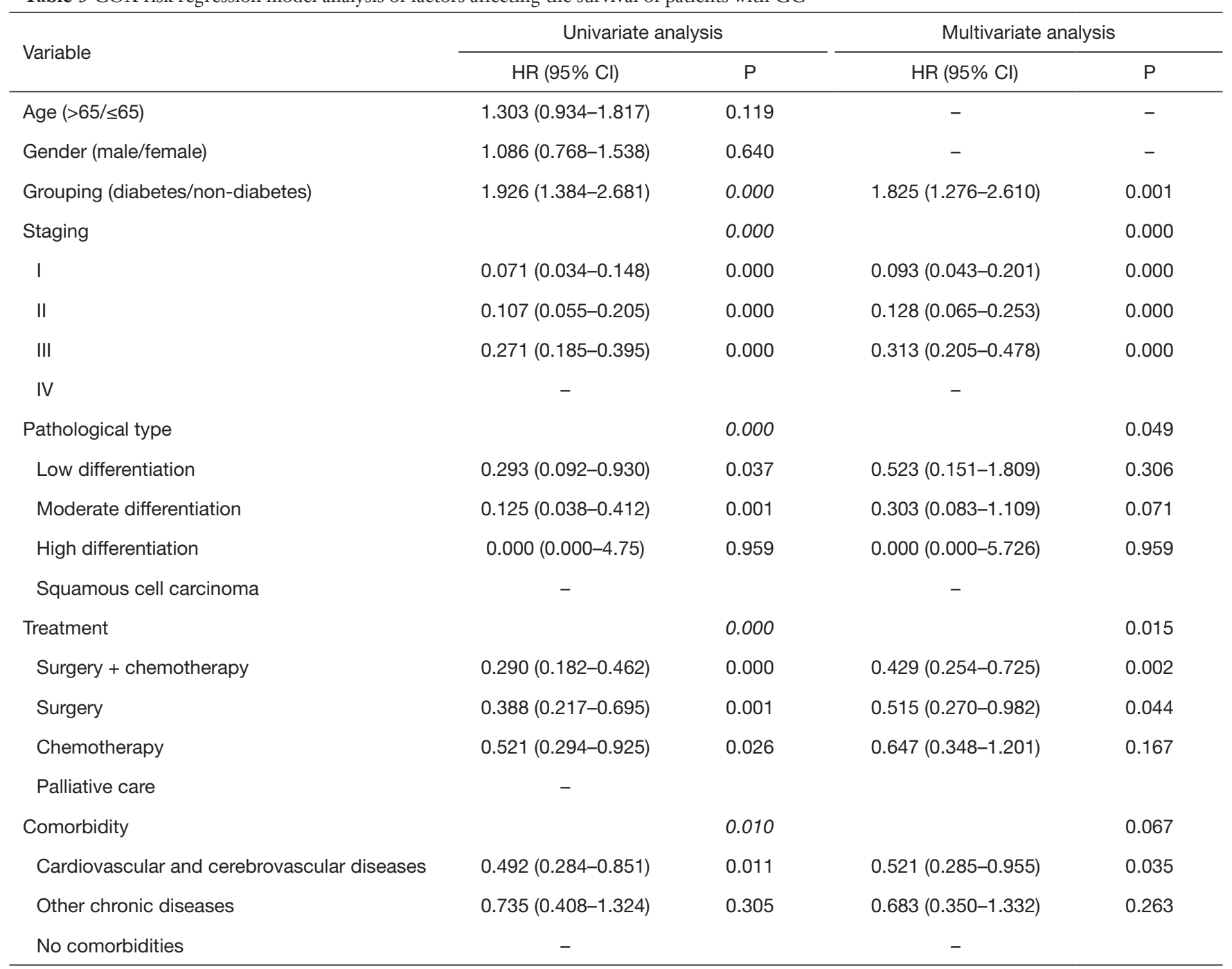

GC, gastric cancer.

Table 4 Analysis of the survival rate of patients with GC before and after matching

\begin{tabular}{|c|c|c|c|c|c|c|}
\hline Survival rate & \multicolumn{3}{|c|}{ Before matching } & \multicolumn{3}{|c|}{ After matching } \\
\hline 1-year survival rate $(\%)$ & 82.1 & 84.4 & 0.639 & 78.6 & 81.0 & 0.735 \\
\hline 3-year survival rate (\%) & 38.1 & 73.1 & 0.000 & 38.1 & 65.5 & 0.004 \\
\hline 5-year survival rate (\%) & 32.1 & 65.6 & 0.000 & 32.1 & 52.4 & 0.023 \\
\hline
\end{tabular}

GC, gastric cancer. 

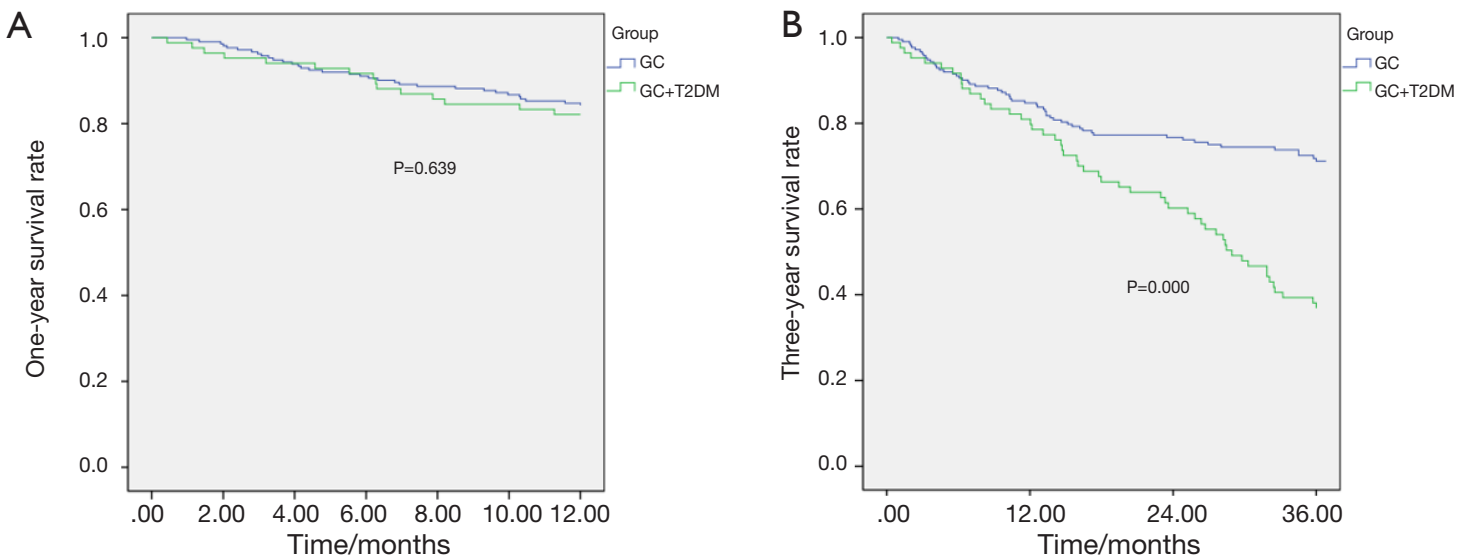

C
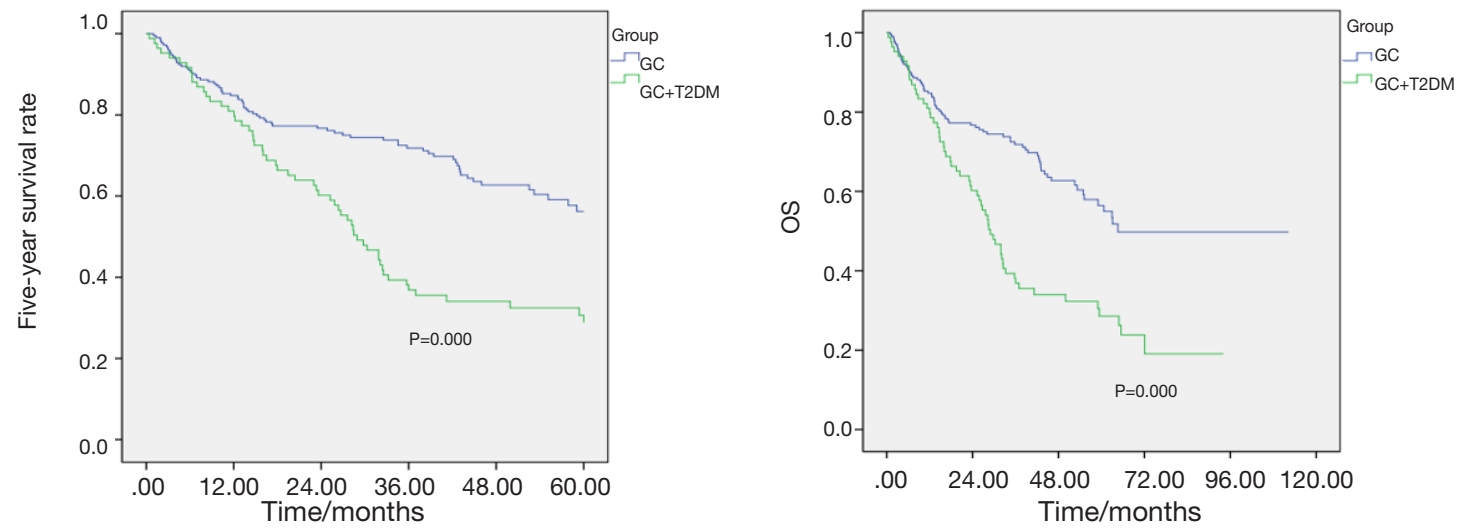

Figure 2 Survival curves of the two groups before the matching. (A) 1-year survival curve of the two groups; (B) 3-year survival curve of the two groups; (C) 5 -year survival curve of the two groups; (D) total survival curve of the two groups.

tissue is an active endocrine organ that produces free fatty acids, interleukin-6, monocyte chemotactic protein, leptin, and other substances, which promote the proliferation, survival, and invasion of cancer cells and inhibit the hosts' autoimmune responses $(18,19)$. Most epidemiological studies have inherent limitations, including their research design, sample size, confusion and bias. Therefore, more elaborate studies should be conducted to explore the link between diabetes and GC, and in-depth institutional research should be performed to explain possible connections.

In this study, the prognostic matching method was used to reduce the confounding and selection biases of the retrospective study, and the balance between the groups in the randomized controlled study was achieved. A total of 215 patients with simple GC were enrolled before the matching, and the clinical data were compared with those of patients with GC and diabetes. Significant differences in age, stage, comorbidities and treatment modalities were observed between the two groups, whereas no comparability was found between the groups. After PSM was completed, only 84 patients with simple GC were included, and the clinical data were compared with those of patients with GC and diabetes. The analysis revealed no significant differences in clinical data across the two groups. The data were thus comparable and could be used for further statistical analysis.

Cox single- and multi-factor analyses were performed on matched patients to examine the factors affecting the survival of patients with GC. Single-factor analysis revealed that grouping, staging, pathological type, treatment, and comorbidities affected the survival of patients with GC. 
A

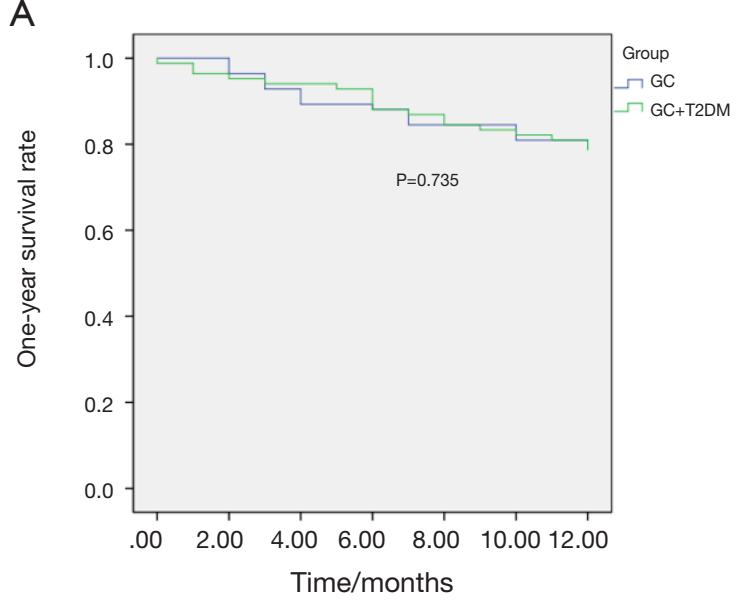

C

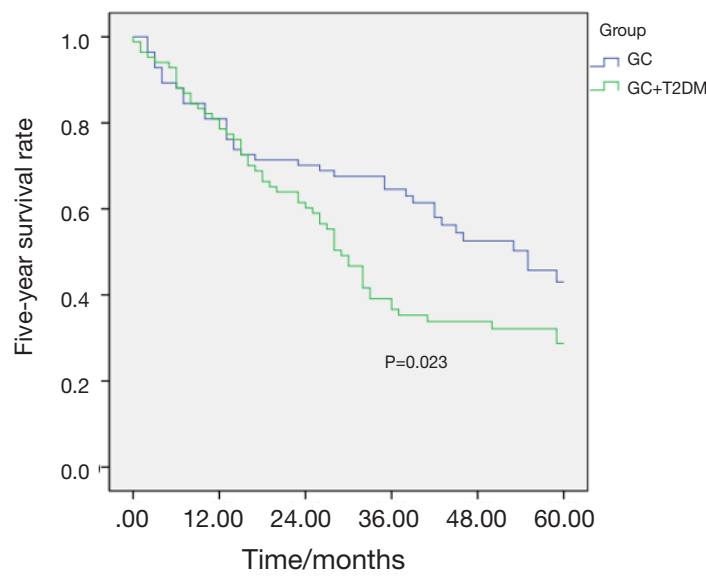

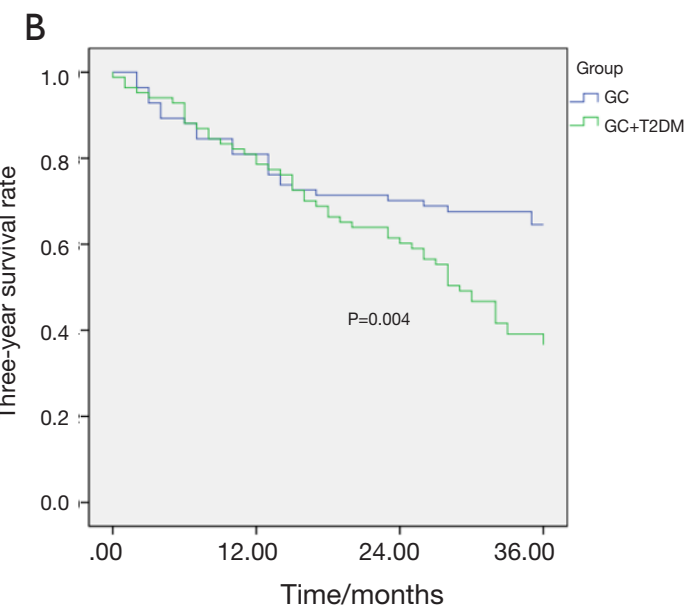

$\mathrm{D}$

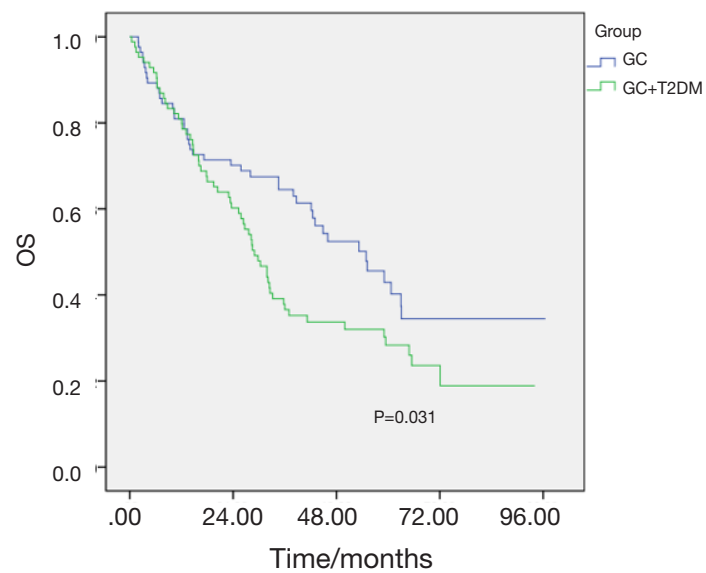

Figure 3 Survival curves of the two groups after matching. (A) 1-year survival curve of the two groups; (B) 3-year survival curve of the two groups; (C) 5-year survival curve of the two groups; (D) total survival curve of the two groups.

Multi-factor analysis found that grouping, pathological type, and treatment were independent risk factors of the survival of patients with GC. The survival of the two groups of subjects was analyzed, with the results indicating that, before matching, the OS rates of patients with GC and those with diabetes were $63.7 \%$ and $28.6 \%$, respectively, which was a statistically significant difference $(\mathrm{P}<0.05)$. The OS rate of patients with GC after matching was the highest (48.8\%). The OS rate of patients with diabetes was $28.6 \%$, and the difference was statistically significant $(\mathrm{P}<0.05)$. These results suggest that the prognosis of patients with GC and diabetes is worse, and that the presence of diabetes indicates a poor prognosis for GC patients with diabetes.

In summary, we were able to confirm that T2DM, which is closely related to the occurrence and development of $\mathrm{GC}$, is one of the factors that can cause the poor prognosis of patients with GC. Clinical treatment should consider whether patients are complicated with T2DM, and timely monitoring should be carried out to avoid disease deterioration.

\section{Acknowledgments}

Funding: Medical Research Development fund of Beijing Kangmeng Charity Foundation YXKY-TB206002.

\section{Footnote}

Reporting Checklist: The authors have completed the 
STROBE reporting checklist. Available at http://dx.doi. org/10.21037/jgo-20-375

Data Sharing Statement: Available at http://dx.doi. org/10.21037/jgo-20-375

Conflicts of Interest: All authors have completed the ICMJE uniform disclosure form (available at http://dx.doi. org/10.21037/jgo-20-375). The authors have no conflicts of interest to declare.

Ethical Statement: The authors are accountable for all aspects of the work in ensuring that questions related to the accuracy or integrity of any part of the work are appropriately investigated and resolved. All procedures performed in this study involving human participants were in accordance with the Declaration of Helsinki (as revised in 2013). The study was approved by Scientific Research and New Technology IRB of Wannnan Medical College Yijinshan Hospital. Because of the retrospective nature of the research, the requirement for informed consent was waived.

Open Access Statement: This is an Open Access article distributed in accordance with the Creative Commons Attribution-NonCommercial-NoDerivs 4.0 International License (CC BY-NC-ND 4.0), which permits the noncommercial replication and distribution of the article with the strict proviso that no changes or edits are made and the original work is properly cited (including links to both the formal publication through the relevant DOI and the license). See: https://creativecommons.org/licenses/by-nc-nd/4.0/.

\section{References}

1. Ferlay J, Soerjomataram I, Dikshit R, et al. Cancer incidence and mortality worldwide: sources, methods and major patterns in GLOBOCAN 2012. Int J Cancer 2015;136:E359-86.

2. Chen W, Zheng R, Zeng H, et al. The incidence and mortality of major cancers in China, 2012. Chin J Cancer 2016;35:73.

3. Bui HDT, Jing X, Lu R, et al. Prevalence of and factors related to microvascular complications in patients with type 2 diabetes mellitus in Tianjin, China: a cross-sectional study. Ann Transl Med 2019;7:325.

4. Sekikawa A, Fukui H, Maruo T, et al. Diabetes mellitus increases the risk of early gastric cancer development. Eur J Cancer 2014;50:2065-71.

5. Qi J, He P, Yao H, et al. Cancer risk among patients with type 2 diabetes: A real-world study in Shanghai, China. J Diabetes 2019;11:878-83.

6. Cheung KS, Chan EW, Chen L, et al. Diabetes Increases Risk of Gastric Cancer After Helicobacter pylori Eradication: A Territory-Wide Study With Propensity Score Analysis. Diabetes Care 2019;42:1769-75.

7. Tseng CH. Diabetes conveys a higher risk of gastric cancer mortality despite an age-standardised decreasing trend in the general population in Taiwan. Gut 2011;60:774-9.

8. Yeh HC, Platz EA, Wang NY, et al. A prospective study of the associations between treated diabetes and cancer outcomes. Diabetes Care 2012;35:113-8.

9. Pan XF, He M, Yu C, et al. Type 2 Diabetes and Risk of Incident Cancer in China: A Prospective Study Among 0.5 Million Chinese Adults. Am J Epidemiol 2018;187:1380-91.

10. Chen Y, Wu F, Saito E, et al. Association between type 2 diabetes and risk of cancer mortality: a pooled analysis of over 771,000 individuals in the Asia Cohort Consortium. Diabetologia 2017;60:1022-32.

11. Hank T, Sandini M, Qadan $M$, et al. Diabetes mellitus is associated with unfavorable pathologic features, increased postoperative mortality, and worse long-term survival in resected pancreatic cancer. Pancreatology 2020;20:125-31.

12. Ge Z, Ben Q, Qian J, et al. Diabetes mellitus and risk of gastric cancer: a systematic review and meta-analysis of observational studies. Eur J Gastroenterol Hepatol 2011;23:1127-35.

13. Shimoyama S. Diabetes mellitus carries a risk of gastric cancer: a meta-analysis. World J Gastroenterol 2013;19:6902-10.

14. Yoon JM, Son KY, Eom CS, et al. Pre-existing diabetes mellitus increases the risk of gastric cancer: a metaanalysis. World J Gastroenterol 2013;19:936-45.

15. Miao ZF, Xu H, Xu YY, et al. Diabetes mellitus and the risk of gastric cancer: a meta-analysis of cohort studies. Oncotarget 2017;8:44881-92.

16. Hua F, Yu JJ, Hu ZW. Diabetes and cancer, common threads and missing links. Cancer Lett 2016;374:54-61.

17. Lega IC, Lipscombe LL. Review: Diabetes, Obesity and Cancer - Pathophysiology and Clinical Implications. Endocr Rev 2020;41:bnz014.

18. Ohara N, Kobayashi M, Ikeda Y, et al. Non-insulin- 
dependent diabetes mellitus induced by immune checkpoint inhibitor therapy in an insulinoma-associated antigen-2 autoantibody-positive patient with advanced gastric cancer. Intern Med 2020;59:551-6.

19. Paternoster S, Falasca M. The intricate relationship

Cite this article as: Sheng L, Peng H, Pan Y, Wang C, Zhu Y. Evaluating the effect of diabetes on the prognosis of gastric cancer using a propensity score matching method. J Gastrointest Oncol 2020;11(5):999-1008. doi: 10.21037/jgo-20375 between diabetes, obesity and pancreatic cancer. Biochim Biophys Acta Rev Cancer 2020;1873:188326.

(English Language Editor: J. Gray) 